\title{
Lepromatous leprosy patients produce antibodies that recognise non-bilayer lipid arrangements containing mycolic acids
}

\author{
Isabel Baeza ${ }^{1 /+}$, Carlos Wong-Baeza ${ }^{1}$, Esther Valerdi', Jeanet Serafin-Lopez ${ }^{2}$, \\ Miguel Ibáñez¹, Sergio Estrada-Parra², Carlos Wong', Iris Estrada-Garcia ${ }^{2}$ \\ 'Biochemistry Department ${ }^{2}$ Immunology Department, National School of Biological Sciences, \\ National Polytechnic Institute, Mexico DF, Mexico
}

Non-bilayer phospholipid arrangements are three-dimensional structures that form when anionic phospholipids with an intermediate structure of the tubular hexagonal phase II are present in a bilayer of lipids. Antibodies that recognise these arrangements have been described in patients with antiphospholipid syndrome and/or systemic lupus erythematosus and in those with preeclampsia; these antibodies have also been documented in an experimental murine model of lupus, in which they are associated with immunopathology. Here, we demonstrate the presence of antibodies against non-bilayer phospholipid arrangements containing mycolic acids in the sera of lepromatous leprosy (LL) patients, but not those of healthy volunteers. The presence of antibodies that recognise these non-bilayer lipid arrangements may contribute to the hypergammaglobulinaemia observed in LL patients. We also found IgM and IgG anti-cardiolipin antibodies in $77 \%$ of the patients. This positive correlation between the anti-mycolic-nonbilayer arrangements and anti-cardiolipin antibodies suggests that both types of antibodies are produced by a common mechanism, as was demonstrated in the experimental murine model of lupus, in which there was a correlation between the anti-non-bilayer phospholipid arrangements and anti-cardiolipin antibodies. Antibodies to non-bilayer lipid arrangements may represent a previously unrecognised pathogenic mechanism in LL and the detection of these antibodies may be a tool for the early diagnosis of LL patients.

Key words: non-bilayer phospholipid arrangements - mycolic acids - lepromatous leprosy

Leprosy is a chronic and degenerative infectious disease caused by Mycobacterium leprae, a Gram-positive and acid-fast bacterium. Recently, the number of cases has decreased considerably as a consequence of opportune detection and effective multidrug treatments. However, the incidence rate (new cases/year) has been stubbornly consistent. The disease can have severe physical and psychological repercussions and it remains a public health problem in Brazil, tropical Africa and Asia (WHO 2010). The clinical signs of leprosy depend on the immunological response of the infected individual. Thus, according to the Ridley-Joplin (1966) classification, leprosy can be characterised by high resistance and low numbers of bacteria [tuberculoid leprosy (TT)] or by low resistance and high numbers of bacteria [lepromatous leprosy (LL)]; between the two ends of this spectrum, borderline tuberculoid (BT) leprosy, borderline leprosy (BB) and borderline lepromatous (BL) leprosy present decreasing resistance and increasing number of bacteria (Ridley \& Jopling 1966). TT is usually limited and can resolve spontaneously without neurological lesions. It can also present with hypopigmented and anaesthetic skin lesions and neurological atrophy, which is caused by immune-

Financial support: IPN Mexico (SIP20110994 to IB, SIP20111083 to CW, SIP20110416 to MI)

+ Corresponding author: ibaeza@encb.ipn.mx

Received 27 March 2012

Accepted 25 July 2012 mediated nerve destruction. Patients develop a strong cellular response, characterised by a Th1 cytokine profile [interleukin (IL)-2, tumour necrosis factor- $\alpha$, interferon (IFN)- $\gamma$ ], macrophage activation by IFN- $\gamma$ (which leads to the killing of intracellular mycobacteria) and normal serum immunoglobulin levels (Murphy et al. 2008). In these cases, lesions show consolidated granulomas with scarce mycobacteria and the lepromin skin test is positive (Britton \& Lockwood 2004).

LL is a systemic and progressive disease that affects all organs (Scollard et al. 2006, Murphy et al. 2008). Even the central nervous system may be involved because $M$. leprae antigens and antibodies against these antigens have been detected in the cerebrospinal fluid of LL patients (Patil et al. 1991, 1995). Moreover, the presence of these antibodies was statistically correlated with upper motor neuron signs in LL patients (Patil et al. 1995). In nodular LL, circumscribed skin lesions (hypopigmented plaques or nodules) are found in auricular pavilions, superciliary regions and limbs. In diffuse LL, patients have generalised and deforming lesions and patients present with a Th2 cytokine profile (IL-4, IL-5, IL-10) and poor cellular responses, which allow intracellular mycobacteria to multiply in macrophages and Schwann cells. Cellular immunity to many antigens is also suppressed and patients develop hypergammaglobulinaemia (Murphy et al. 2008); immune complexes obstruct blood capillaries and lead to an inflammatory response (via complement fixation and neutrophil recruitment) that causes tissue damage (Nath et al. 2000, Prabhu et al. 2009); mycobacteria are abundant in nasal mucosa, skin lesions and blood and the lepromin skin test is negative. BB has the 
clinical and immunological characteristics of TT and LL; BT leprosy is lepromin-positive and BL leprosy is lepromin-negative. According to the World Health Organisation (WHO) operational classification (WHO 1982), TT and BT leprosy are considered paucibacillary (PB) and usually present with five or fewer skin lesions and only one affected nerve, whereas LL, BL leprosy and $\mathrm{BB}$ are multibacillary and usually present with more than five skin lesions and more than one affected nerve (WHO 1982, Britton \& Lockwood 2004).

Over the last 12 years, several groups have investigated the presence of antiphospholipid antibodies in leprosy (Santiago et al. 1989). Interestingly, the antiphospholipid antibodies in leprosy are similar to those in patients suffering from antiphospholipid syndrome, but of a different isotype, with IgM being more prevalent (de Larrañaga et al. 2000). Thus, leprosy-related antiphospholipid antibodies comprise persistent IgG and IgM anti-beta 2 glycoprotein I ( $\beta_{2}$ GPI), which differ from antiphospholipid syndromerelated antibodies with respect to IgG subclass, avidity and epitope specificity, possibly reflecting a distinct pathophysiological significance (Arvieux et al. 2002).

Anti-cardiolipin antibodies are often present in leprosy $(\sim 50 \%)$ and they are frequently associated with the presence of anti- $\beta_{2}$ GPI antibodies (44.8\%), although they do not appear to be correlated with thromboembolic events (Escobar-Gutiérrez et al. 1990, Levy et al. 2004, Fenniche et al. 2007, Sène et al. 2008). However, thromboembolic events in LL patients have recently been associated with thalidomide or corticosteroid treatment (Vetrichevvel et al. 2008, Yamaguchi et al. 2012). In addition, LL patients may also present with thromboembolic events associated with single nucleotide polymorphisms of the $\beta_{2}$ GPI gene, such as Leu247Val and Trp326Ser (Brochado et al. 2010). Until now, the specificity of the antiphospholipid antibodies produced by LL patients and, in particular, LL patients have not been studied in detail.

Lipids constitute more than $40 \%$ of the dry weight of mycobacteria (Goren \& Brennan 1979). The cellular membrane of $M$. leprae contains two characteristic lipopolysaccharides (lipoarabinomannan and lipomannan), whereas the cell wall consists of peptidoglycan covalently bound to arabinogalactan, and mycolic acids (MAs). The capsule contains a high level of mycobacterium-specific lipids, which are related to virulence and antibiotic resistance, including phthiocerol dimycocerosate, trehalose mycolates (including chord factor), trehalose sulpholipids (SL), phenolic glycolipid-1 (PGL-1) and MAs (Brennan \& Barrow 1980, Britton et al. 2000, $\mathrm{Ng}$ et al. 2000, Rao et al. 2006, Scollard et al. 2006).

MAs are long fatty acids (60-90 carbon atoms) composed of a shorter beta-hydroxy chain with a longer alpha-alkyl side chain; the number of carbons varies by species. There are three main types of MAs: alpha, methoxy and keto. Alpha-MAs comprise at least $70 \%$ of the MAs present in mycobacteria and contain several cyclopropane rings; methoxy-MAs with several methoxy groups comprise between $10-15 \%$ of the MAs and the remaining $10-15 \%$ are keto-MAs, which contain several ketone groups (Minnikin \& Goodfellow 1980, Besra \& Chatterjee 1994, Barry et al. 1998, Rao et al. 2006,
Brennan \& Crick 2007). Considering the abundance of mycobacteria in the organs of LL patients $\left(3 \times 10^{10} / \mathrm{g}\right.$ of tissue) (Waters et al. 1978), it is not surprising that some antibodies are directed against lipid-rich mycobacterial antigens. Moreover, lipid-rich mycobacterial molecules released from dead mycobacteria could easily interact with host molecules to create neoantigens, which could, in turn, induce the production of antibodies.

Of particular interest are the long hydrocarbon MAs because, based on their chemical structure, we propose that they are amphiphilic molecules with a conical molecular shape (the cross-sections of their hydrophobic regions are larger than those of their hydrophilic regions) and that this conical molecular shape suggests that MAs might have a role as lipid mycobacterial antigens. Cationic molecules with a conical molecular shape, such as the drugs chlorpromazine (CPZ) and procainamide, can induce the formation of intermediate structures of the hexagonal phase II $\left(\mathrm{H}_{\mathrm{II}}\right)$ arrangements by some anionic conic phospholipids, including phosphatidic acid (PA), phosphatidylserine and cardiolipin; these intermediate forms of $\mathrm{H}_{\mathrm{II}}$ arrangements are inserted into the bilayer arrangement of the other phospholipids and distort it to form non-bilayer phospholipid arrangements or lipidic particles (Aguilar et al. 1999, Baeza et al. 2004, Wong-Baeza et al. 2012). Furthermore, when injected intrasplenically in mice, non-bilayer phospholipid arrangements induce the production of specific antibodies and antiphospholipid antibodies that recognise non-bilayer phospholipid arrangements have been reported in patients with antiphospholipid syndrome (Baeza et al. 2004) and patients with preeclampsia (Campos et al. 2006). Here, we report that MAs induce the formation of non-bilayer lipid arrangements in liposomes (model membranes) and that $83 \%$ of patients with LL have antibodies that specifically bind to these non-bilayer lipid arrangements containing MAs; moreover, $77 \%$ of these patients also have IgM and $\operatorname{IgG}$ anti-cardiolipin antibodies. Antiphospholipid antibodies against non-bilayer lipid arrangements may be relevant to the pathogenesis of leprosy.

\section{SUBJECTS, MATERIALS AND METHODS}

Patients - Thirty untreated LL patients with a confirmed diagnosis and a bacterial index range of $1+$ to 4+ (age range 15-73 years) were included in this study; their diagnoses were established according to the guidelines of the Mexican Ministry of Health, which are based on the Ridley-Jopling classification (Ridley \& Jopling 1966). A further 30 healthy volunteers were also included in this study for comparison purposes. Venous blood $(5 \mathrm{~mL})$ was collected in anticoagulant-free tubes and centrifuged at 2,600 $\mathrm{g}$ for $10 \mathrm{~min}$. Serum was stored in aliquots of $250 \mu \mathrm{L}$ at $-70^{\circ} \mathrm{C}$ until antibody detection by ELISA or flow cytometry. Patients and healthy volunteers signed an informed consent form; the local ethical committees of the institutions involved approved this protocol in accordance with international guidelines.

Preparation of liposomes and liposomes with nonbilayer phospholipid arrangements - Unilamellar liposomes were prepared using the reverse-phase evaporation method (Szoka \& Papahadjopoulos 1978) that was modified (Baeza et al. 1994). The following lipids and lipid 
mixtures were dissolved in $1 \mathrm{~mL}$ of chloroform (Mallinckrodt Baker, Phillipsburg, NJ, USA), $3 \mu \mathrm{mol}$ of phosphatidylcholine (PC), $2 \mu \mathrm{mol}$ of PC and $1 \mu \mathrm{mol}$ of PA (2:1 molar ratio), $2 \mu \mathrm{mol}$ of PC and $1 \mu \mathrm{mol}$ of MAs (2:1 molar ratio), and $2 \mu \mathrm{mol}$ of PC, $0.5 \mu \mathrm{mol}$ of PA and $0.5 \mu \mathrm{mol}$ of MAs (2:0.5:0.5 molar ratio). All lipids were from SigmaAldrich (St. Louis, MO, USA) and they were at least 97\% pure. The MAs (Cat. M 4537) were a mixture of long (78-90 carbon residues) branched beta-hydroxy acids, molecular weight range of 1,108-1,307 Da, that were obtained from the cell wall of Mycobacterium tuberculosis $(M t b)$ (human strain). Each lipid solution was placed in a two-neck glass flask connected to a vacuum pump and chloroform was evaporated at reduced pressure. Next, $1 \mathrm{~mL}$ of diethyl ether (Mallinckrodt Baker, Phillipsburg, NJ, USA) and $330 \mu \mathrm{L}$ of TS buffer (Tris- $\mathrm{HCl} 10 \mathrm{mM}$, $\mathrm{NaCl} 1 \mathrm{mM}, \mathrm{pH} 7.0$ ) were added and the mixtures were mixed in a vortex and sonicated three times ( 5 sec sonication followed by $30 \mathrm{sec}$ resting period) using a Lab Supply G112SPI sonicator (Laboratory supplies, Hicksville, NY, USA). The diethyl ether was then removed under a stream of oxygen-free dry nitrogen at reduced pressure using a rotary evaporator at $37^{\circ} \mathrm{C}$. During this process, the mixtures changed from viscous gels to suspensions. To complete the $1 \mathrm{~mL}$ of liposome suspension, $600 \mu \mathrm{L}$ of TS buffer was added and the suspension was filtered through a Millipore membrane (Billerica, MA, USA) with $0.45-\mu \mathrm{m}$ pores to homogenise the liposome size and maintain sterility (Baeza et al. 1994).

Where indicated, the inductors of non-bilayer phospholipid arrangements, $\mathrm{Mn}^{2+}\left(5 \mathrm{mM} \mathrm{MnCl}_{2}\right)$ and $\mathrm{CPZ}(3$ $\mathrm{mM}$ ) (all from Sigma-Aldrich), were added to the liposome suspensions, which were incubated at $37^{\circ} \mathrm{C}$ for 30 min to induce the formation of non-bilayer phospholipid arrangements (Baeza et al. 1994, 2004).

Liposome suspensions, with or without non-bilayer phospholipid arrangements, were analysed by flow cytometry (FACSCalibur, Becton Dickinson, San José, CA, USA) (Aguilar et al. 1999, Baeza et al. 2004); 10,000 events were acquired per sample and the data were analysed using CellQuest software (Becton Dickinson). The dot plots of size [forward scatter vs. granularity side scatter (SSC)] and histograms of granularity (SSC) are shown. The Kolmogorov-Smirnov test was used to compare the granularity histogram of each sample with the negative control (liposome suspension without $\mathrm{MnCl}_{2}$ or $\mathrm{CPZ}$ ); $\mathrm{D} \geq 0.5$ with $\mathrm{p} \leq 0.001$ was considered significant and revealed the presence of non-bilayer phospholipid arrangements in the liposome membranes (Aguilar et al. 1999, Baeza et al. 2004, Wong-Baeza et al. 2012).

Detection of antibodies that bind non-bilayer lipid arrangements in sera - Flow cytometry was used to detect antiphospholipid antibodies in the patients' sera that bind non-bilayer lipid arrangements. Liposomes made from PC/PA/MAs or PC/PA ( $0.1 \mu \mathrm{mol}$ of conic lipids in $100 \mu \mathrm{L}$ of TS buffer), treated with $5 \mathrm{mM} \mathrm{MnCl}$, were mixed with $100 \mu \mathrm{L}$ of serum (from LL patients or healthy volunteers) and incubated for $1 \mathrm{~h}$ at $37^{\circ} \mathrm{C}$ (the sera were previously heated at $56^{\circ} \mathrm{C}$ for $30 \mathrm{~min}$ to inactivate complement and diluted 1:50 in blocking solution). After incubation, $100 \mu \mathrm{L}$ of fluorescein isothiocyanate (FITC)-labelled goat anti-human polyvalent antibody (Sigma-Aldrich) diluted 1:2000 in blocking solution was added. After $1 \mathrm{~h}$ at $37^{\circ} \mathrm{C}$, TS solution was added up to a volume of $1 \mathrm{~mL}$ and the samples were analysed by flow cytometry as described above. A monoclonal antibody (H-308) that binds non-bilayer phospholipid arrangements was used as a positive control (Aguilar et al. 1999, Baeza et al. 2004). The percentage of positive events in each sample was determined. The results from the 30 patients were compared with the results from the 30 healthy volunteers using the Kruskal-Wallis test with Dunn's post-test (the threshold for significance was set at $\mathrm{p} \leq 0.05$ ) and the KolmogorovSmirnov test was used to compare the fluorescence-1 histogram of each patient with the negative control (liposome suspension with $5 \mathrm{mM} \mathrm{MnCl}$ treatment stained with FITC-labelled goat anti-human polyvalent antibody); $\mathrm{D} \geq 0.5$ with $\mathrm{p} \leq 0.001$ was considered significant and indicated the presence of antibodies that bind non-bilayer lipid arrangements in the sera analysed.

Detection of antibodies that bind non-bilayer phospholipid arrangements by liposomal ELISA - The ability of the antiphospholipid antibodies in the sera to bind liposomes with non-bilayer phospholipid arrangements was also assessed using the liposomal ELISA method (Aguilar et al. 1999, Wong-Baeza et al. 2012). An aliquot of 100 $\mu \mathrm{L}$ of PC/PA liposome suspension, with or without $5 \mathrm{mM}$ $\mathrm{MnCl}_{2}$ treatment, was added to each well of a 96-well flatbottom plate (Costar Co, Cambridge, MA, USA) and left at room temperature (RT) for $12 \mathrm{~h}$. The plate was decanted and $200 \mu \mathrm{L}$ of blocking solution ( $8 \%$ foetal bovine serum in TS buffer) was added to each well. After $1 \mathrm{~h}$ at RT, the plate was decanted and $100 \mu \mathrm{L}$ of serum (from LL patients or healthy volunteers) was added to each well and left for $1 \mathrm{~h}$ at $37^{\circ} \mathrm{C}$ (the sera were previously heated at $56^{\circ} \mathrm{C}$ for 30 min to inactivate complement and diluted 1:250 in blocking solution). The plate was washed five times with 200 $\mu \mathrm{L}$ of blocking solution per well and then $100 \mu \mathrm{L}$ of goat anti-human polyvalent antibody conjugated to peroxidase (Sigma-Aldrich) and diluted 1:2000 in blocking solution was added to each well. After $1 \mathrm{~h}$ at $37^{\circ} \mathrm{C}$, the plate was washed five times with $200 \mu \mathrm{L}$ of blocking solution per well and $100 \mu \mathrm{L}$ of peroxidase substrate solution $(10 \mathrm{mg}$ of o-phenylenediamine and $20 \mu \mathrm{L}$ of $30 \% \mathrm{H}_{2} \mathrm{O}_{2}$ in $25 \mathrm{~mL}$ TS buffer) was added to each well. After $20 \mathrm{~min}$ at $37^{\circ} \mathrm{C}$, the enzymatic reaction was stopped by the addition of $50 \mu \mathrm{L}$ of sulphuric acid $2.5 \mathrm{M}$ to each well and the absorbance at $492 \mathrm{~nm}$ was measured in a Labsystems Multiskan MS photometric plate reader (MTX Labsystems, Vienna, Virginia, USA). The monoclonal antibody (H-308) against the non-bilayer phospholipid arrangements was used as a positive control (Aguilar et al. 1999, Baeza et al. 2004); the negative control was a liposome suspension with $5 \mathrm{mM}$ $\mathrm{MnCl}_{2}$ treated with goat anti-human polyvalent antibody conjugated to peroxidase. The results from the 30 patients were compared with the results from the 30 healthy volunteers using the Kruskal-Wallis test with Dunn's post-test (significance was set at $\mathrm{p} \leq 0.05$ ) and the mean absorbance of the healthy volunteers plus two standard deviations was set as the threshold for positive values, indicating the presence of antibodies that bind non-bilayer lipid arrangements in the sera analysed. 
Detection of anti-cardiolipin antibodies by ELISA Anti-cardiolipin antibodies were measured using the anticardiolipin $\operatorname{IgG} / \operatorname{IgM}$ enzyme immunoassay test kit from Diamedix (IVAX Diagnostics, Miami, FL, USA) according to the manufacturer's instructions. This kit enables the semi-quantitative detection of $\operatorname{IgG}$ and IgM antibodies to cardiolipin in human serum using a 96-well ELISA plate coated with highly purified bovine cardiolipin and saturated using highly purified human $\beta_{2} \mathrm{GPI}$, which is a cofactor required for the binding of anti-cardiolipin antibodies. The results are reported in GPL or MPL units per mL, where 1 GPL unit is the binding activity of $1 \mu \mathrm{g} / \mathrm{mL}$ of a reference $\operatorname{IgG}$ anti-cardiolipin antibody and 1 MPL unit is the binding activity of $1 \mu \mathrm{g} / \mathrm{mL}$ of a reference IgM anticardiolipin antibody (Harris et al. 1987). The results from the $30 \mathrm{LL}$ patients were compared with the results from the 30 healthy volunteers using the Kruskal-Wallis test with Dunn's post-test (significance was set at $p \leq 0.05$ ). In accordance with the manufacturer's instructions, results of less than 10 GPL or MPL units per $\mathrm{mL}$ were considered negative, while results of 12 or more GPL or MPL units per $\mathrm{mL}$ were considered positive for the presence of $\operatorname{IgG}$ or IgM anti-cardiolipin antibodies, respectively.

\section{RESULTS}

MAs induce the formation of non-bilayer phospholipid arrangements on liposomes - PC, which is composed of a choline head group, glycerophosphoric acid, a saturated fatty acid (such as stearic acid) and an unsaturated fatty acid (such as oleic acid), has a cylindrical molecular shape because the cross-section of its hydrophobic region is similar to that of its hydrophilic region (Fig. 1A). However, PA, which is composed of a glycerophosphoric acid, a saturated fatty acid and an unsaturated fatty acid, has a conical molecular shape because the cross-section of its hydrophobic region is larger than that of its hydrophilic region (Fig. 1B). When liposomes made of these phospholipids are suspended in a buffer devoid of inductors of non-bilayer phospholipid arrangements, such as divalent cations, their lipid bilayer is smooth, which is consistent with low granularity. In the presence of $\mathrm{Mn}^{2+}$, PA molecules form intermediate structures of the $\mathrm{H}_{\text {II }}$ tridimensional arrangements within the bilayers formed by PC molecules; these structures are known as non-bilayer phospholipid arrangements or lipidic particles and they can be detected as liposome granularity increases (Aguilar et al. 1999, Baeza et al. 2004, Wong-Baeza et al. 2012). Consistent with these findings, the liposomes containing a conic phospholipid, such as those made of PC/PA, have increased granularity in the presence of $\mathrm{Mn}^{2+}$ (Fig. 2A); however, the granularity of the liposomes made of PC alone is not altered in the presence of $\mathrm{Mn}^{2+}$ (Fig. 2B) because, in this case, no conic phospholipids are available to form the lipidic particles. Cationic drugs with a conical molecular shape, such as CPZ, can also form lipidic particles with PA (Baeza et al. 2004, Wong-Baeza et al. 2012) and accordingly, liposomes made of PC/PA have increased granularity in the presence of CPZ (Fig. 2C). Taking into account the molecular form of phospholipids and their molecular arrangements, we proposed that MAs can also have a conical molecular shape (Fig. 1C, D) be- cause they are fatty acids with long hydrocarbon chains (60-90 carbon residues) and also branched beta-hydroxy acids with cyclopropane, methoxy or ketone groups (Minnikin \& Goodfellow 1980, Besra \& Chatterjee 1994, Barry et al. 1998, Rao et al. 2006). We further hypothesised that they could induce the formation of non-bilayer lipid arrangements on liposomes (Fig. 1E). The addition of MAs to PC or PC/PA liposomes increases the granularity (Fig. 2E, F) and the granularity is further increased in the presence of $\mathrm{Mn}^{2+}$ (Fig. 2D-F) $(\mathrm{D} \geq 0.5$ with $\mathrm{p} \leq$ 0.001 , compared with the granularity histogram of liposomes without $\mathrm{Mn}^{2+}$ ), which strongly suggests that nonbilayer lipid arrangements containing MAs are formed. Similar results to those shown in Fig. 2E were obtained using $3 \mathrm{mM} \mathrm{Ca}^{2+}$ (Supplementary data). A slight increase in liposome size was also observed (Fig. 2D-F) because the presence of non-bilayer phospholipid arrangements promotes membrane fusion (Baeza et al. 2004).

$L L$ patients produce antibodies that recognise nonbilayer lipid arrangements containing MAs - The sera of the LL patients, but not those of the healthy volunteers, contain antibodies that recognise non-bilayer phospholipid arrangements on PC/PA liposomes (Fig. 3A, B) and importantly also on PC/PA/MA liposomes (Fig. 3C). These antibodies were detected by flow cytometry (Fig. 3A, C) and

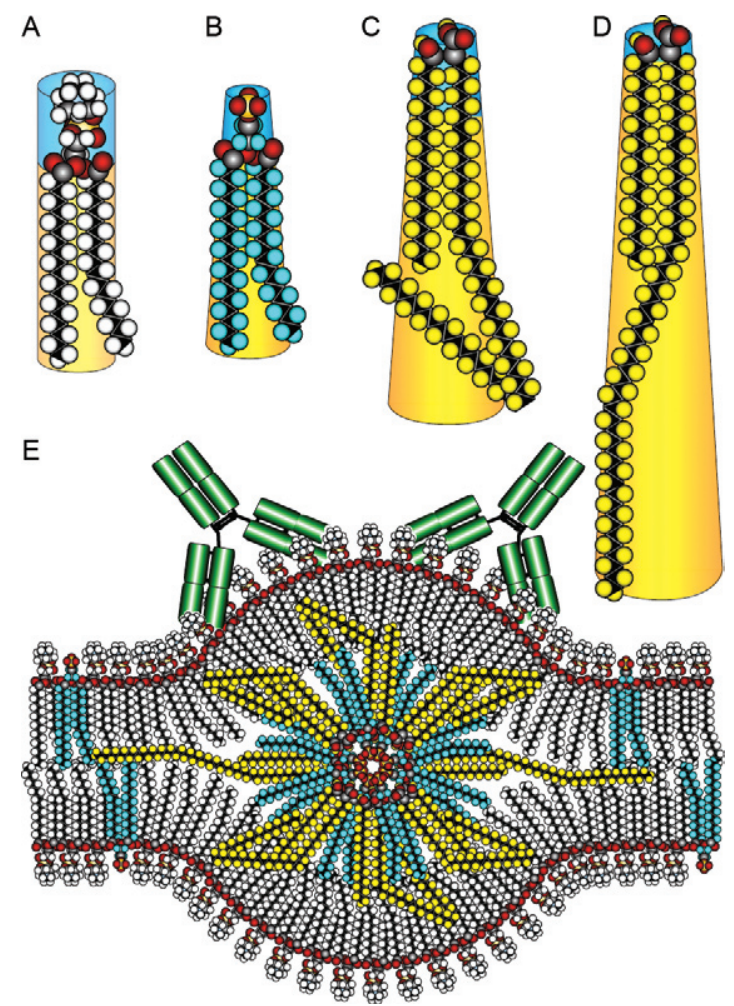

Fig. 1: molecular organization of the non-bilayer lipid arrangement containing alpha-mycolic acids. Phosphatidylcholine (A) has a cylindrical molecular shape, while phosphatidic acid (B) and alpha-mycolic acids (C, D) are conic molecules. The inverted micelle formed by phosphatidic and alpha-mycolic acids is inserted in the bilayer arrangement of the liposome, forming the non-bilayer lipid arrangement. 
liposomal ELISA (Fig. 3B). Amongst the 30 LL patients tested by flow-cytometry, using PC/PA liposomes bearing non-bilayer phospholipid arrangements, 29 (97\%) had values of $D \geq 0.5$ with $p \leq 0.001$ when compared with the negative control (liposomes with non-bilayer phospholipid arrangements stained with FITC-labelled goat anti-human polyvalent antibody); 28 of the 30 healthy volunteers had values of $\mathrm{D} \leq 0.05$ with this same assay. These results indicate the presence of antibodies against non-bilayer phospholipid arrangements in the LL patients and the lack of these antibodies in the healthy volunteers. Amongst the 30 LL patients tested by flow-cytometry, using PC/PA/ MAs liposomes bearing non-bilayer lipid arrangements,

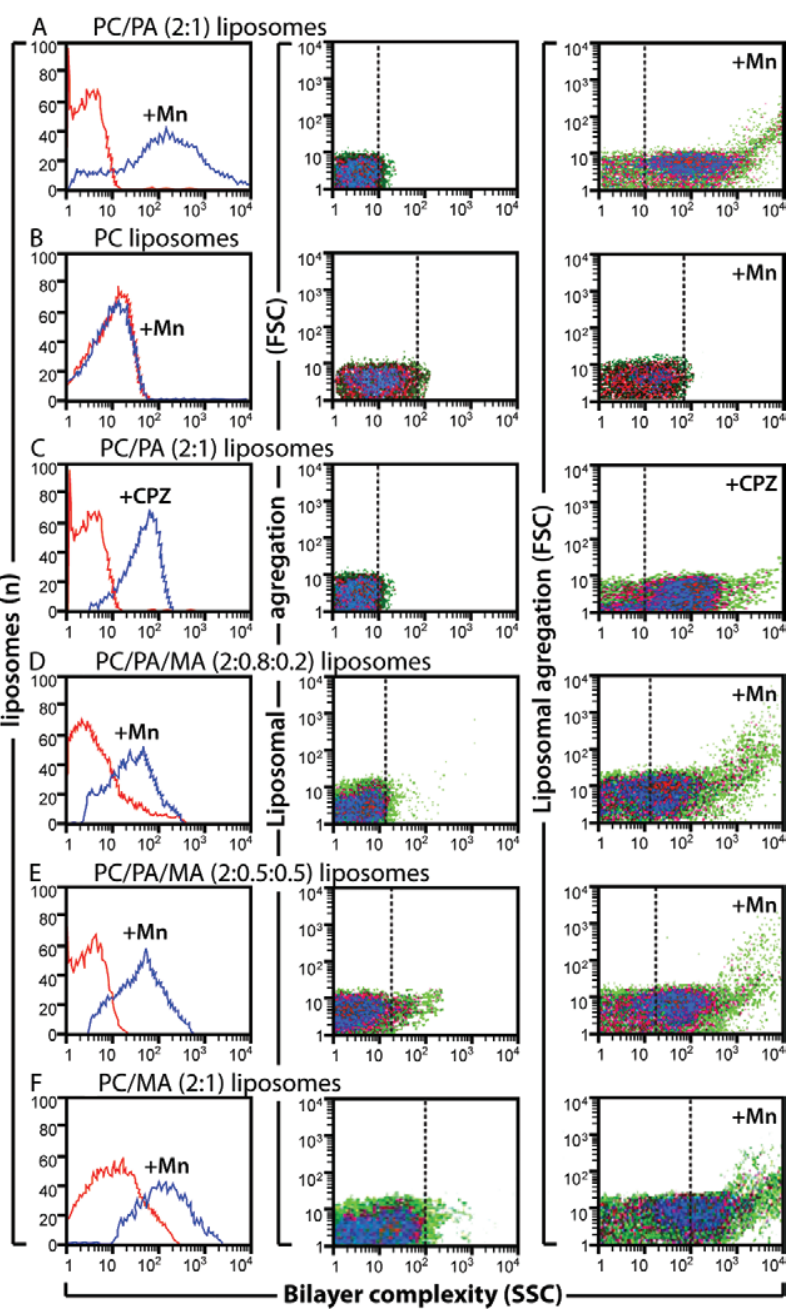

Fig. 2: detection of non-bilayer lipid arrangements on liposomes by flow cytometry. Liposomes made of phosphatidylcholine (PC)/phosphatidic acid (PA) (2:1 molar ratio), PC, PC/PA/mycolic acid (MA) (2:0.8:0.2), PC/PA/MA (2:0.5:0.5) or PC/MA (2:1) were incubated at $37^{\circ} \mathrm{C}$ for 30 min with $5 \mathrm{mM} \mathrm{MnCl}$, or with $3 \mathrm{mM}$ chlorpromazine (CPZ). Changes in bilayer complexity [side scatter (SSC)] and liposomal aggregation [forward scatter (FSC)] were evaluated. Bilayer complexity is represented in histograms (red lines represent liposomes alone, blue lines represent liposomes with $\mathrm{Mn}^{2+}$ or with CPZ); bilayer complexity and aggregation of liposomes alone or with $\mathrm{Mn}^{2+}$ or $\mathrm{CPZ}$ are represented in density plots. Broken lines indicate reference values for changes in SSC for bilayer complexity.
25 (83\%) had values of $\mathrm{D} \geq 0.5$ with $\mathrm{p} \leq 0.001$ compared with the negative control. All of the healthy volunteers had values of $\mathrm{D} \leq 0.05$, indicating the presence of antibodies against the non-bilayer lipid arrangements containing MAs in the LL patients and the lack of these antibodies in the healthy volunteers. Of the 30 serum samples with the highest antibody titres against the non-bilayer phospholipid arrangements according to the liposomal-ELISA method, eight were tested by immune adsorption assay (Supplementary data). Additionally, the levels of antibodies against non-bilayer lipid arrangements containing MAs are 4.4 times lower than those against non-bilayer phospholipid arrangements containing PA. All of the LL patients tested by liposomal ELISA had absorbance values above the positive threshold (0.09), which confirmed the
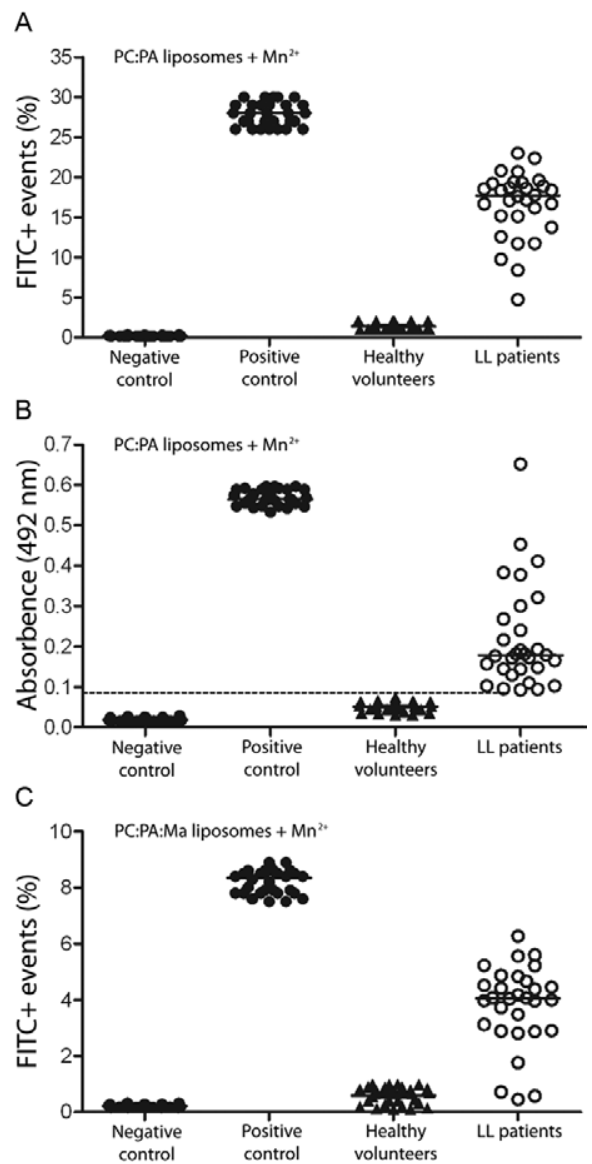

Fig. 3: sera of lepromatous leprosy (LL) patients bind specifically to non-bilayer lipid arrangements. The sera of LL patients and of healthy volunteers were assayed by flow cytometry $(\mathrm{A}, \mathrm{C})$ or by liposomal ELISA (B) using $\mathrm{Mn}^{2+}$-treated phosphatidylcholine (PC)/phosphatidic acid (PA) liposomes or $\mathrm{Mn}^{2+}$-treated $\mathrm{PC} / \mathrm{PA} /$ mycolic acid (MA) liposomes. Data from 30 patients were compared with those from 30 healthy volunteers using Kruskal-Wallis test with Dunn's post-test (significance was set at $\mathrm{p} \leq 0.001$ in $\mathrm{A}$ and at $\mathrm{p} \leq 0.05$ in $\mathrm{C}$ ) and the mean absorbance of the healthy volunteers plus two standard deviations was set as the threshold for positive values. $\mathrm{Mn}^{2+}$-treated liposomes incubated with fluorescein isothiocyanate (FITC)-labelled goat anti-human polyvalent antibodies or with the monoclonal antibody (H-308) that binds non-bilayer phospholipid arrangements were used as the negative and positive control, respectively. 
presence of antibodies that recognise non-bilayer phospholipid arrangements in the sera of these patients.

The binding of the monoclonal antibody H-308, which is specific to non-bilayer phospholipid arrangements (Aguilar et al. 1999, Baeza et al. 2004, Wong-Baeza et al. 2012), to PC/PA liposomes bearing non-bilayer phospholipid arrangements or to $\mathrm{PC} / \mathrm{PA} / \mathrm{MA}$ liposomes bearing non-bilayer lipid arrangements, was used as a positive control in the flow cytometry and liposomal ELISA studies (Fig. 3A-C).

The levels of antibodies that recognise non-bilayer phospholipid arrangements are correlated with the levels of anti-cardiolipin antibodies in LL patients - Amongst the $30 \mathrm{LL}$ patients, $23(77 \%)$ were positive for IgG anticardiolipin antibodies (Fig. 4A) and 23 of these same LL patients $(77 \%)$ were positive for IgM anti-cardiolipin antibodies (Fig. 4B). The levels of both the IgM and IgG anti-cardiolipin antibodies were positively correlated with the levels of antibodies that recognise non-bilayer phospholipid arrangements (measured by ELISA) in the sera of LL patients (Fig. 4C, D).

\section{DISCUSSION}

Non-bilayer phospholipid arrangements are transiently formed on cellular membranes, where they participate in several physiological processes, such as membrane fusion (Hafez et al. 2000, Hafez \& Cullis 2001, Wong-Baeza et al. 2010), the organisation of tight junctions (Wegener \& Galla 1996) and the activation of membrane enzymes (van den Brink-van der Laan et al. 2001).
However, if they are stabilised, as occurs in the presence of divalent cations, such as $\mathrm{Ca}^{2+}$ and $\mathrm{Mn}^{2+}$ or drugs like CPZ and procainamide, they induce the production of specific antiphospholipid antibodies (Baeza et al. 2004, Wong-Baeza et al. 2012). Antiphospholipid antibodies that recognise non-bilayer phospholipid arrangements have been described in patients with antiphospholipid syndrome and/or systemic lupus erythematosus (these patients also have anti-cardiolipin antibodies) (Baeza et al. 2004) and in patients with preeclampsia (Campos et al. 2006). These antibodies have also been documented in an experimental model of lupus in BALB/c and NIH mice, in which they are associated with immunopathology (Baeza et al. 2004, Wong-Baeza et al. 2012).

In this study, we demonstrate that MAs, which we propose have a conical molecular shape similar to that of the phospholipid PA, induce the formation of nonbilayer lipid arrangements on liposomes, as assessed by increased liposome granularity and by the reactivity of these lipid arrangements containing MAs with the monoclonal antibody H-308. This antibody specifically recognises non-bilayer phospholipid arrangements and does not show any reactivity with cardiolipin immobilised on solid supports, such as the ELISA plates used in the diagnosis kits to clinically measure antiphospholipid antibodies (Baeza et al. 2004, 2011). A combination of these two experimental approaches led us to conclude that MAs induce the formation of non-bilayer lipid arrangements on the liposomes studied. Furthermore, the composition of the liposomes used (PC and PA in a 2:1
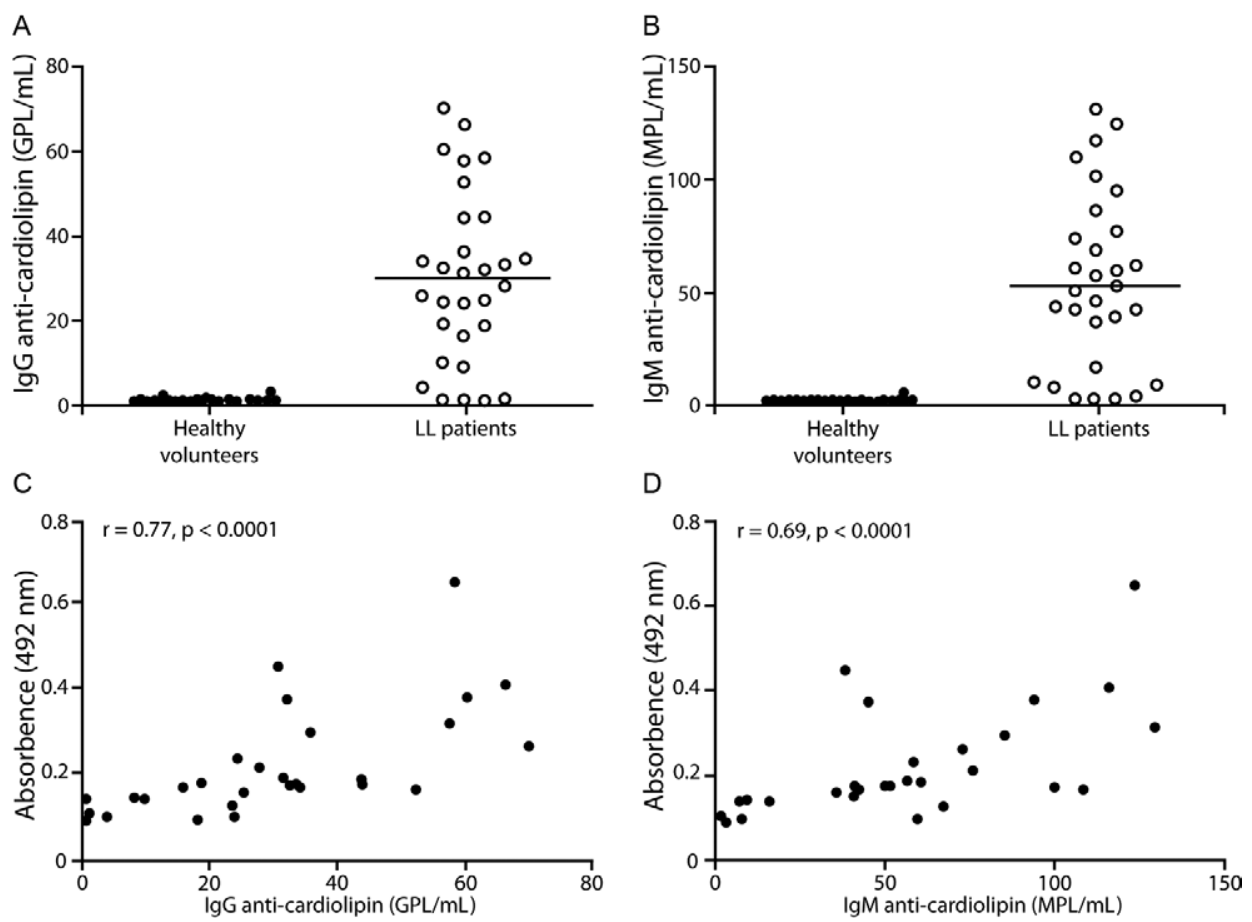

Fig. 4: the levels of antibodies that recognise non-bilayer phospholipid arrangements correlate with the units of anti-cardiolipin antibodies in lepromatous leprosy (LL) patients. The units of $\operatorname{IgG}(\mathrm{A})$ and $\operatorname{IgM}(\mathrm{B})$ anti-cardiolipin antibodies were measured by ELISA in the serums of LL patients, data from 30 patients were compared with data from 30 healthy volunteers using Kruskal-Wallis test with Dunn's post-test (significance was set at $\mathrm{p} \leq 0.05$ ). The units of $\operatorname{IgG}$ and IgM anti-cardiolipin antibodies showed positive correlations with the levels of antibodies that recognize non-bilayer phospholipid arrangements (measured by ELISA) in the serums of LL patients (C, D). 
molar ratio) closely resembles that of cellular membranes (Voet \& Voet 2011) and $\mathrm{Mn}^{2+}$, the inducer of non-bilayer lipid arrangements, is a trace element present at concentrations of $87.2 \pm 17.8 \mathrm{ng} / \mathrm{dL}$ in the sera of the control subjects (Yilmaz et al. 2005). Moreover, tissues of LL patients can have very high levels of mycobacteria (3.3 x $10^{10} / \mathrm{g}$ of tissue) (Waters et al. 1978) and because lipids constitute more than $40 \%$ of the dry weight of mycobacteria (Goren \& Brennan 1979), the amount of MAs released from dead mycobacteria in these tissues would be considerable. Therefore, it is possible that non-bilayer lipid arrangements containing MAs, similar to those induced by MAs on liposomes, may be formed in the cellular membranes of LL patients. Cations, such as $\mathrm{Mn}^{2+}$ and $\mathrm{Ca}^{2+}$ released from lysed cells and damaged tissues, could further increase the number of these non-bilayer lipid arrangements. Considering the complexity of the mycobacterial cell wall, it seems improbable that free MAs could form non-bilayer lipid arrangements in the mycobacterial cell membrane. Furthermore, we found that the addition of MAs to liposomes in molar ratios higher than 1.0 caused the production of irregular aggregates and liposomes of smaller size (data not shown), which suggests that MAs at high concentrations could disrupt cellular membranes. These findings suggest that MAs, in addition to their recognised role in the survival and pathogenesis of mycobacteria, may play other important roles in the physiopathology of LL.

The presence of antibodies that recognise non-bilayer lipid arrangements in LL patients, but not in healthy volunteers, was demonstrated using two complementary methods: ELISA and flow cytometry. We performed immune adsorption assays in the serum samples that had the highest antibody titres against non-bilayer phospholipid arrangements using the ELISA method. Of the 30 serum samples from the LL patients, eight were analysed in this way. The titres of antibodies against non-bilayer lipid arrangements containing MAs did not change after immune adsorption on ELISA plates coated with cardiolipin. No data regarding anti-PGL-1 antibodies are available for any of the sera used here because this test is not used by health authorities in Mexico. Rojas-Espinosa et al., in 1999, showed that Mexican patients have no specific recognition for M. leprae PGL-1 or Mtb SL-I, ruling out the possibility of using PGL-I or SL-I as tools for the differential diagnosis of these two mycobacterial diseases.

In the LL sera, the levels of antibodies against PC/PA liposomes were higher than those against PC/PA/MA liposomes. This difference could be attributed to the irregular molecular form of MAs (1 of their hydrocarbon chain is shorter than the other). The non-bilayer lipid arrangements that they form might be more irregular, making them less immunoreactive than those arrangements formed by PA. However, these antibodies could have activities similar to those described in our experimental mouse model of lupus triggered by non-bilayer phospholipid arrangements. These mice develop facial alopecia and symmetric malar masks with deposits of immune complexes, extensive epidermal atrophy, a mild inflammatory infiltrate and decreased terminal hair fol- licles, which present a disaggregation of matricial cells surrounded by an inflammatory infiltrate and haemorrhage (Baeza et al. 2004, Wong-Baeza et al. 2012). Even with their lower titres, the antibodies against non-bilayer lipid arrangements containing MAs could cause skin lesions, such as vasculitis and necrosis, which have been described in the Lucio phenomenon, a variant of LL found in Mexico (Monteiro et al. 2012).

Antibodies that recognise non-bilayer lipid arrangements may contribute to the hypergammaglobulinaemia that is observed in LL patients. Moreover, these antibodies may bind to non-bilayer lipid arrangements stabilised by MAs on cellular membranes, which would lead to complement fixation and cellular lysis as well as an inflammatory response that would increase tissue damage. The release of intracellular contents could promote the production of antibodies that recognise molecules not usually found in the extracellular space, such as cardiolipin (Baeza et al. 2004, Wong-Baeza et al. 2012), which is a constituent of mitochondrial inner membranes. Previous studies (Santiago et al. 1989, Escobar-Gutiérrez et al. 1990, Levy et al. 2004) report anti-cardiolipin antibodies in almost $50 \%$ of 55 patients with LL and here we report IgM and IgG anti-cardiolipin antibodies in $77 \%$ of the $30 \mathrm{LL}$ patients tested. It is noteworthy that a higher percent of these patients had antibodies that recognise non-bilayer phospholipid arrangements (100\% using ELISA) because this could indicate that anti-cardiolipin antibodies are formed at later stages of the pathology.

The positive correlation that is observed between the antibodies that recognise non-bilayer phospholipid arrangements and the anti-cardiolipin antibodies further suggests that both types of antibodies are produced in LL patients through a common mechanism, as demonstrated in the experimental mouse model of lupus (Baeza et al. 2004, Wong-Baeza et al. 2012). In this murine model, the liposomes with non-bilayer phospholipid arrangements induce the production of antibodies that specifically bind these arrangements in mice; these antibodies induce cellular damage, which exposes several autoantigens such as cardiolipin, histones, nuclear proteins and DNA, thereby inducing the production of autoantibodies. Interestingly, in this murine model, the antibodies that bind non-bilayer phospholipid arrangements appear four weeks before the anti-cardiolipin, anti-histone and lupus anticoagulant antibodies, which demonstrate, at least in this pathology, a positive correlation between the antibodies that recognise non-bilayer phospholipid arrangements and the anti-cardiolipin, anti-histones and lupus anticoagulant antibodies.

Our finding that MAs induce the formation of nonbilayer lipid arrangements, which are recognised by antibodies present in the sera of the LL patients, suggests that in these patients the antibodies may trigger the events described in the murine model of lupus and contribute to the pathology of leprosy. The antibodies that bind to these arrangements may represent a previously unrecognised pathogenic mechanism during LL. In addition, we found that the titres of the antibodies that bind non-bilayer phospholipid arrangements containing MAs and those that bind cardiolipin were highest amongst the LL patients that had a bacterial index range close to $4+$. 
Renal lesions have been reported in $72 \%$ of a cohort of Brazilian leprosy patients, including patients with erythema nodosum leprosy and LL. These patients present with amyloidosis, glomerulonephritis, nephrosclerosis and other renal lesions; glomerular lesions can be caused by immune complexes and they mainly occur during the reactional states of erythema nodosum leprosy (Nakayama et al. 2001, Prabhu et al. 2009). This information is relevant to our experimental murine model of lupus in which we have described kidney abnormalities consisting of diffuse cellular proliferation in the glomeruli and immune complex deposits along the basal membranes of the glomerular capillaries and in the mesangial matrix (Baeza et al. 2004, Wong-Baeza et al. 2012). The antibodies against non-bilayer lipid arrangements detected in LL patients may be involved in the development of the kidney lesions in leprosy.

We reported high titres of antibodies against nonbilayer phospholipid arrangements in our experimental murine model that resembles human lupus as well as in 35 patients who meet four or more of the American Rheumatism Association criteria for systemic lupus erythematosus; in both cases, these antibodies were strongly correlated with the development of disease (Baeza et al. 2004, 2011, Wong-Baeza et al. 2012). In addition, 38 patients with hypertensive pregnancy disorders (preeclampsia or eclampsia) were positive for these antibodies, although their serum titres were lower than those of the lupus patients. The patients with preeclampsia/eclampsia had increased non-bilayer phospholipid arrangements in the syncytiotrophoblast, extravillous cytotrophoblast, syncytial knots and the amnion epithelial cell membranes of the placenta. The authors of this study concluded that the non-bilayer phospholipid arrangements and their antibodies were one of the pathogenic factors associated with these pregnancy pathologies (Campos et al. 2006). The titres of the antibodies that bind non-bilayer phospholipid arrangements were lowest in the LL patients compared with those in the lupus patients and the preeclampsia/ eclampsia patients. However, these antibodies may have pathogenic activities similar to those described in the skin and subcutaneous tissue and in the glomeruli of our experimental murine model of human lupus.

Further studies are required to confirm the presence of non-bilayer lipid arrangements containing MAs in tissue samples from LL patients. Further assessment of the presence of antibodies that recognise non-bilayer lipid arrangements and of anti-cardiolipin antibodies at different time points during the disease is also necessary. The study of PB leprosy patients, both tuberculoid and BT, as well as patients with other infectious diseases, such as tuberculosis, is important. Patients with PB leprosy and latent tuberculosis may have low titres of antibodies against non-bilayer lipid arrangements containing MAs because both diseases are characterised by the presence of few mycobacteria, which are mostly included in granulomas.

The efficient diagnosis of leprosy is the subject of ongoing research. The use of anti-PGL-1 antibodies has been proposed as an adjunct early leprosy diagnostic marker for household contacts of patients with leprosy
(Bazan-Furine et al. 2011). The histopathological examination of nerve samples, in conjunction with clinical, epidemiological and laboratory data, has also been investigated for use in the diagnosis of pure neural leprosy (Antunes et al. 2012). Because antibodies that bind nonbilayer lipid arrangements may have pathogenic activity in the development of skin and renal lesions, they may be a useful tool for the early diagnosis of LL.

\section{ACKNOWLEDGEMENTS}

To Dr Luis España, for assisting in some flow cytometry experiments, to Dr Cristina Carreño, for assisting with clinical identification of leprosy patients, and to Dr Isabel WongBaeza, for discussion and assistance in the preparation of the English manuscript.

\section{REFERENCES}

Aguilar L, Ortega-Pierres G, Campos B, Fonseca R, Ibáñez M, Wong C, Farfán N, Naciff JM, Kaetzel MA, Dedman JR, Baeza I 1999. Phospholipid membranes form specific nonbilayer molecular arrangements that are antigenic. J Biol Chem 274: 25193-25196.

Antunes SLG, Chimelli L, Jardim MR, Vital RT, Nery JAC, CorteReal S, Hacker MAVB, Sarno EN 2012. Histopathological examination of nerve samples from pure neural leprosy patients: obtaining maximum information to improve diagnostic efficiency. Mem Inst Oswaldo Cruz 107: 246-253.

Arvieux J, Renaudineau Y, Mane I, Perraut R, Krilis SA, Youinou P 2002. Distinguishing features of anti-beta 2 glycoprotein I antibodies between patients with leprosy and the antiphospholipid syndrome. Thromb Haemost 87: 599-605.

Baeza I, Ibáñez M, Wong C 2011. Method for antiphospholipid syndrome. United States Patent USA 7,867,723.

Baeza I, Leyva E, Campos B, Lara M, Ibáñez M, Farfán N, Orozco H, Flores-Romo L, Hernández-Pando R, Wong C 2004. Antibodies to nonbilayer phospholipid arrangements induce a murine autoimmune disease resembling human lupus. Eur J Immunol 34: 576-586.

Baeza I, Wong C, Ibáñez M, Mondragón R, González S, Farfán N, Argüello C 1994. Transbilayer diffusion of divalent cations mediated by phosphatidate particles. J Mol Evol 39: 560-568.

Barry CE, Lee RE, Mdluli K, Sampson AE, Schroeder BG, Slayden RA, Yuan Y 1998. Mycolic acids: structure, biosynthesis and physiological function. Prog Lipid Res 37: 143-179.

Bazan-Furini R, Motta ACF, Simao JCL, Tarquínio DC, Marques Jr W, Barbosa MHN, Foss NT 2011. Early detection of leprosy by examination of household contacts, determination of serum anti-PGL-1 antibodies consanguinity. Mem Inst Oswaldo Cruz 106: 536-540.

Besra GS, Chatterjee D 1994. Lipids and carbohydrates of Mycobacterium tuberculosis. In BR Bloom, Tuberculosis pathogenesis, protection and control, American Society for Microbiology, Washington DC, p. 285-306.

Brennan PJ, Barrow W 1980. Evidence of species-specific lipid antigens in Mycobacterium leprae. Int J Lepr 49: 382-387.

Brennan PJ, Crick DC 2007. Structure of cell wall of Mycobacterium. Curr Topics Med Chem 7: 475-488.

Britton WJ, Lockwood DNJ 2004. Leprosy. Lancet 363: 1209-1219.

Britton WJ, Martin E, Kamath AT, Neupane KD, Roche PW 2000. Immunoprophylaxis against Mycobacterium leprae infection with subunit vaccines. Lepr Rev 71: 176-181.

Brochado MJ, Figueiredo JF, Mendes-Junior CT, Louzada-Junior P, Kim OM, Roselino AM 2010. Correlation between beta-2-gly- 
coprotein I gene polymorphism and anti-beta-2 glycoprotein I antibodies in patients with multibacillary leprosy. Arch Dermatol Res 302: 583-591.

Campos B, Chames M, Lantry JM, Bill JP, Eis A, Brockman D, Neil J, Tischner E, Barton J, Wong C, Schwemberger S, Cornelius J, Myatt L, Baeza I, Hnat M 2006. Determination of non-bilayer phospholipid arrangements and their antibodies in placentae and sera of patients with hypertensive disorders of pregnancy. Placenta 27: 215-224.

de Larrañaga GF, Forastiero RR, Martinuzzo ME, Carreras LO, Tsariktsian G, Sturno MM, Alonso BS 2000. High prevalence of antiphospholipid antibodies in leprosy: evaluation of antigen reactivity. Lupus 9: 594-600.

Escobar-Gutiérrez A, Amezcua-Chavarria ME, Pastén S, Castro E, Flores O, Rodríguez O 1990. Anti-cardiolipin antibodies in Mexican lepromatous leprosy patients. Int J Lepr 58: 723-724.

Fenniche S, Benmously R, Sfia M, Daoud L, Debbiche A, Ben Ayed M, Mokhtar I 2007. Late-occurring cutaneous vasculitis after successful treatment of diffuse lepromatous leprosy: Lucio's phenomenon. Med Trop (Mars) 67: 65-68.

Goren MB, Brennan PJ 1979. Mycobacterium lipids: chemistry and biological activities. In GP Youmans, Tuberculosis, Saunders WB Co, Philadelphia, p. 63-193.

Hafez IM, Ansell S, Cullis PR 2000. Tunable pH-sensitive liposomes composed of mixtures of cationic and anionic lipids. Biophys $J$ 79: $1438-1446$.

Hafez IM, Cullis PR 2001. Roles of lipid polymorphism in intracelular delivery. Adv Drug Deliv Rev 47: 139-148.

Harris EN, Gharavi AE, Patel S, Hughes GRV 1987. Evaluation of the anticardiolipin antibody test: report of an international workshop held April 4, 1986. Clin Exp Immunol 68: 215-222.

Levy RA, de Meis E, Pierangeli S 2004. An adapted ELISA method for differentiating pathogenic from nonpathogenic aPL by a beta 2 glycoprotein I dependency anticardiolipin assay. Thromb Res 114: 573-577.

Minnikin DE, Goodfellow M 1980. Lipid composition in the classification and identification of acid-fast bacteria. Soc Appl Bacteriol Symp Ser 8: 189-256.

Monteiro R, de Abreu MA, Tiezzi MG, Roncada EV, de Oliveira CC, Ortigosa LC 2012. Lucio's phenomenon: another case reported in Brazil. An bras Dermatol 87: 296-300.

Murphy K, Travers P, Walport M 2008. Janeway's immunobiology, 7th ed., Garland Science, New York, 504 pp.

Nakayama EE, Ura S, Fleury RN, Soares V 2001. Renal lesions in leprosy: a retrospective study of 199 autopsies. Am J Kidney Dis 38: 26-30.

Nath I, Vemuri N, Reddi AL, Jain S, Brooks P, Colston MJ, Misra RS, Ramesh V 2000. The effect of antigen presenting cells on the cytokine profiles of stable and reactional lepromatous leprosy patients. Immunol Lett 75: 69-76.

Ng V, Zanazzi G, Timpl R, Talts J, Salzer JL, Brennan PJ, Rambukkana A 2000. Role of the cell wall phenolic glycolipid-1 in the peripheral nerve predilection of Mycobacterium leprae. Cell 103: 511-529.

Patil SA, Katoch K, Ramu G, Sengupta U 1995. Detection of antibodies against phenolic glycolipid-1 (PGL-1), 35-kDa and 30-40-kDa components of Mycobacterium leprae in the cerebrospinal fluid of leprosy patients. J Med Microbiol 43: 115-159.

Patil SA, Tyagi P, Katoch K, Sreevatsa, Sengupta U 1991. Antigens of Mycobacterium leprae in the cerebrospinal fluid of leprosy patients: detection by monoclonal-antibody-based sandwich immunoradiometric assay and avidin/biotin immunoblotting. Clin Exp Immunol 84: 515-521.
Prabhu S, Shenoi SD, Pai SB, Sripathi H 2009. Erythema nodosum leprosum as the presenting feature in multibacillary leprosy. Dermatol Online J 15: 15-18.

Rao V, Gao F, Chen B, Jacobs Jr WR, Glickman MS 2006. Transcyclopropanation of mycolic acids on trehalose dimycolate suppresses Mycobacterium tuberculosis-inflammation and virulence. J Clin Invest 116: 1660-1667.

Ridley DS, Jopling WH 1966. Classification of leprosy according to immunity. A five-group system. Int J Lepr Other Mycobact Dis 34: 255-273.

Rojas-Espinosa O, Luna-Herrera J, Arce-Paredes P 1999. Recognition of phenolic glycolipid-I (Mycobacterium leprae) and sulfolipid-I (M. tuberculosis) by serum from Mexican patients with leprosy or tuberculosis. Int J Tuberc Lung Dis 3: 1106-1112.

Santiago MB, Cossermelli W, Tuma MF, Pinto MN, Oliveira RM 1989. Anticardiolipin antibodies in patients with infectious diseases. Clin Rheumatol 8: 23-28.

Scollard DM, Adams LB, Gillis TP, Krahenbuhl JL, Truman RW, Williams DL 2006. The continuing challenges of leprosy. Clin Microbiol Rev 19: 338-381.

Sène D, Piette JC, Cacoub P 2008. Antiphospholipid antibodies, antiphospholipid syndrome and infections. Autoimmun Rev 7: 272-277.

Szoka Jr F, Papahadjopoulos D 1978. Procedure for preparation of liposomes with large internal aqueous space and high capture by reverse-phase evaporation. Proc Natl Acad Sci USA 75: 4194-4198.

van den Brink-van der Laan E, Dalbey RE, Demel RA, Killian JA, de Kruijff B 2001. Effect of nonbilayer lipids on membrane binding and insertion of catalytic domain of leader peptidase. Biochemistry 40: 9677-9684.

Vetrichevvel TP, Pise GA, Thappa DM 2008. A case report of venous thrombosis in a leprosy patient treated with corticosteroid and thalidomide. Lepr Rev 79: 193-195.

Voet D, Voet JG 2011. Biochemistry, 4th ed., John Wiley \& Sons Inc, New York, 219 pp.

Waters MF, Bakri IB, Isa HJ, Rees RJ, McDougall AC 1978. Experimental lepromatous leprosy in the white-handed gibbon (Hylobatus lar): successful inoculation with leprosy bacilli of human origin. Br J Exp Pathol 59: 551-557.

Wegener J, Galla HJ 1996. The role of non-lamellar lipid structures in the formation of tight junctions. Chem Phys Lipids 81: 229-255.

WHO - World Health Organization 1982. Chemotherapy of leprosy for control programmes. WHO Tech Rep Ser 675: 1-33.

WHO - World Health Organization 2010. Global leprosy situation 2010. Wkly Epidemiol Rec 85: 337-348.

Wong-Baeza C, Bustos I, Serna M, Tescucano A, Alcántara-Farfán V, Ibáñez M, Montañez C, Wong C, Baeza I 2010. Membrane fusion inducers, chloroquine and spermidine increase lipoplex-mediated gene transfection. Biochem Biophys Res Commun 396: 549-554.

Wong-Baeza C, Hernández-Pando R, Reséndiz A, Tescucano A, Bustos I, Ibáñez M, Wong C, Baeza I 2012. Molecular organization of the non-bilayer phospholipid arrangements that induce an autoimmune disease resembling human lupus in mice. Mol Memb Biol 29: 52-67.

Yamaguchi S, Yamamoto Y, Hosokawa A, Hagiwara K, Uezato H, Takahashi K 2012. Deep venous thrombosis and pulmonary embolism secondary to co-administration of thalidomide and oral corticosteroid in a patient with leprosy. J Dermatol 39: 711-714.

Yilmaz A, Sari RA, Gundogdu M, Kose N, Dag E 2005. Trace elements and some extracelular antioxidant protein levels in serum of patients with systemic lupus erythematosus. Clin Rheumatol 24: 331-335. 


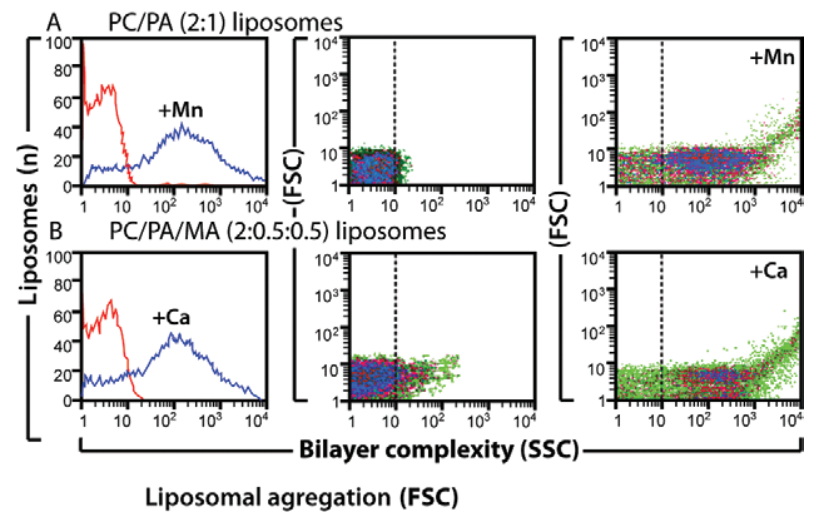

Detection of non-bilayer lipid arrangements on liposomes by flow cytometry. Liposomes made of phosphatidylcholine (PC)/phosphatidic acid (PA) $\left(2: 1\right.$ molar ratio) or PC/PA/mycolic acid (MA) $(2: 0.5: 0.5)$ were incubated at $37^{\circ} \mathrm{C}$ for 30 min with $5 \mathrm{mM} \mathrm{MnCl2}$ or with $3 \mathrm{mM} \mathrm{CaCl2}$. Changes in bilayer complexity [side scatter (SSC)] and liposomal aggregation [forward scatter (FSC)] were evaluated. Bilayer complexity is represented in histograms (red lines represent liposomes alone, blue lines represent liposomes with $\mathrm{Mn}^{+}$or $\mathrm{Ca}^{+}$); bilayer complexity and aggregation of liposomes alone or with $\mathrm{Mn}^{+}$or $\mathrm{Ca}_{2}^{+}$are represented in density plots.

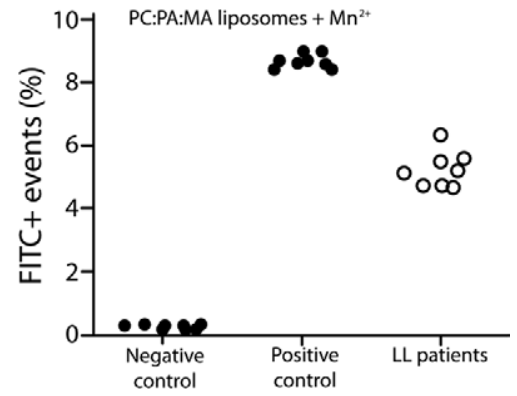

Sera of lepromatous leprosy (LL) patients bind specifically to non-bilayer lipid arrangements. Eight sera of LL patients were assayed by flow cytometry using Mn2+-treated phosphatidylcholine (PC)/phosphatidic acid (PA)/mycolic acid (MA) liposomes as antigens. The eight sera were previously immune adsorbed using cardiolipin-coated ELISA plates (Baeza et al. 2004). Mn2+-treated liposomes incubated with fluorescein isothiocyanate (FITC)-labelled goat anti-human polyvalent antibody or with the monoclonal antibody (H-308) that binds non-bilayer phospholipid arrangements were used as the negative and positive control, respectively. Immune adsorption assays were made as was previously described. Sera samples at 1:50 dilution in $8 \%$ foetal bovine serum in TS buffer were added to cardiolipin-coated ELISA plates. After $1 \mathrm{~h}$ incubation at $37^{\circ} \mathrm{C}$, sera were removed and incubated again in new cardiolipin-coated ELISA plates, a procedure repeated three times to achieve complete anti-cardiolipin antibodies adsorption. 